\title{
Parental decision about human papillomavirus vaccination for their daughters
}

\author{
CHENG LS Winnie \\ Tung Wah College, \\ Hong Kong \\ Email: winniecheng@twc.edu.hk
}

\begin{abstract}
Background: Human papillomavirus (HPV) infection is a sexually transmitted infection common in teenagers and adults in their early 20s. Some types of HPV are associated with $90 \%$ of cervical cancer around the world. The risk of HPV infection in women can be reduced by giving HPV vaccine to girls beginning at the age of 9, as recommended by Centers for Disease Control and Prevention (CDC). For children and young adolescents, parental decision on HPV vaccination is crucial for the uptake of HPV vaccine rate. This study explored the factors influencing parental decisions on HPV vaccination for their daughters. Methods: A cross-sectional survey was conducted between February and March 2017. Data were collected from parents of girls aged 9 to 17 ( $\mathrm{n}=420,100 \%$ response rate) in Hong Kong. A validated 27-item questionnaire was used to elicit parents' knowledge on HPV and HPV vaccine, barriers to HPV vaccination, and willingness toward vaccinating their daughter(s). Results: Only $59.6 \%$ of respondents were aware of the HPV vaccine before this study. Among the parents who were aware of the vaccine, around $32 \%$ had already vaccinated their daughters. Majority $(83 \%)$ of the parents had heard of HPV, but the knowledge levels were poor (70\% scored below average). The reasons parents did not vaccinate their daughters were fear of the side-effects of the vaccine $(85.7 \%)$ and feared the vaccine would affect the immune system of their daughters $(38.5 \%)$; around $18 \%$ believed that HPV vaccination would encourage early sexual activity or promiscuity. More than half of the respondents $(69.7 \%)$ were willing to vaccine their daughter(s). Conclusions: Given the evidence of suboptimal awareness about HPV vaccination, education and promotional strategies should be enhanced. Interventions should address parental concerns about the misconceptions between vaccination and early sexual activity and promiscuity.
\end{abstract}

Keywords- Human papillomavirus (HPV), parental decision, HPV vaccine, sexually transmitted infection (STI), cervical cancer

\section{INTRODUCTION}

Human papillomavirus (HPV) infection is a sexually transmitted infection common in teenagers and adults in their early 20s [1]. Some types of HPV are associated with $90 \%$ of anogenital warts and around $70 \%$ of cervical cancers [2]. HPVs infect both males and females through intimate sexual contact [1]. The more sexual partners, the higher is the risk of HPV infection [3]. In Hong Kong, among people who took the cervical screening test, the HPV infection prevalence rate was $7 \%$ to $11 \%$ [4]. To lower the risk of infection, HPV vaccination is recommended by Centers for Disease Control and Prevention [1]. The risk of HPV infection in women can be reduced by giving HPV vaccine to girls beginning at the age of 9 [5]

A systemic review of studies over 10 years revealed that the HPV vaccine reduced the risk of some types of HPV infection by up to $90 \%$ [6]. Evidence showed that the HPV vaccine could substantially reduce the incidence of cervical precancerous lesions in young girls by $70.2 \%$ and effective in lowering the risk of cervical cancer [7]. To combat the risk of HPV infection, a Free Cervical Cancer Vaccination Pilot Scheme was launched as the first step in introducing the HPV vaccine into immunization programs for girls aged 9 to 18 in Hong Kong [8]

Despite the significant effect of the HPV vaccine, the uptake rate of vaccination in Hong Kong is relatively low. One study reported that only $7.2 \%$ of young adolescent girls received the HPV vaccine in 2013 [9]. Probably, vaccine uptake is determined by parental decision, in particular children and young adolescents. As children aged 9-17 are still too young to make such health decisions and usually obey parents; parental knowledge about HPV or HPV vaccine may be a factor for HPV vaccination of their daughters [9, 10, 11].

In Hong Kong, there is a paucity of research on parental knowledge of HPV and their willingness for the vaccination of their children. This study sought to explore the factors influencing parental decisions on HPV vaccination for their daughters. The results can contribute to the understanding the barriers leading to low vaccination coverage in Hong Kong, and thus provide a foundation for tailoring promotion strategies The current study attempted to address the following research questions: 
1. What is the knowledge about and willingness towards HPV vaccination for daughters among Hong Kong parents?

2. Is there any relationship between knowledge and parental willingness towards HPV vaccination for daughters among Hong Kong parents?

\section{METHODS}

\section{A. Design and sampling}

A descriptive survey using questionnaires was conducted in February and March 2017. Sample size was estimated based on the total number of Hong Kong domestic households from June to August 2016, which was 2,508,400 [12]. A sample of 385 subjects was required to have desired marginal error within $5 \%$ and confidence level at $95 \%$. A total number of 420 participants was recruited to cover $10 \%$ attrition rate. The study was carried out in four different districts in Hong Kong. Convenience sampling was used to recruit parents attending the student health service centres. Inclusion criteria were (1) Hong Kong residents, and (2) parents with at least one daughter aged 9 to 17. Parents who cannot read Chinese were excluded from our study.

\section{B. Data collection}

The data was collected using a questionnaire modified from a previous study with similar objectives in Texas [13]. The Questionnaire contains 22 items in three areas: (1) knowledge about HPV and HPV infection (7 items), the knowledge score was assessed ranging from 0 to 7 . A higher knowledge score indicated better knowledge of HPV and HPV infection. (2) barriers that affect parental willingness towards vaccination including cost, risk perception, perception of relationship with early sexual behavior, and media reports (14 items); (3) willingness towards HPV vaccination for their daughter(s) (1 item). Additional information on demographic data was included to assess age, educational level and family monthly income.

To ensure the questionnaire is culturally relevant, it was translated into Chinese with forward and backward processes. The original questionnaire was forward-translated into Chinese by researchers. Backward translation was done by a bilingual research specialist residing in America. Congruency check was done by a bilingual professor residing in England. The resultant Chinese version of the questionnaire was tested twice with 20 participants for stability of the instrument. The correlation coefficient was 0.791 , indicating the stability of the questionnaire. Internal consistency was high with Cronbach alpha at 0.937 .

\section{ETHICAL CONSIDERATIONS}

Our study was approved by the College Ethics Committee in Tung Wah College. An information sheet with details about the study purpose, procedure, confidentiality, risk and benefit was provided to each participant. Their consent was obtained before data collection. The participants were assured that all information would be kept strictly confidential and used for research purpose only.

\section{DATA ANALYSIS}

Data collected were analyzed by the Computerized Statistical Package of Social Science, version 23. Descriptive statistics were used to analyze demographic data. Pearson correlation was computed to test the association between knowledge of HPV and willingness toward HPV vaccination. In all statistical analyses, a $p$-value of less than 0.05 was considered as statistical significant.

\section{RESUlTS}

Four hundred and twenty parents participated in this study. After checking of data, 413 valid questionnaires were analyzed.

\section{A. Demographic data}

Three hundred $(73 \%)$ participants were mothers and 113 were fathers. About $50 \%$ of both mothers and fathers had attained secondary education, and around $25 \%$ had attained bachelor and higher degrees. A majority (around 80\%) of the parents were aged below 50 . Nearly $68 \%$ of the families had monthly income of HK\$20,000 (US\$2600) or above. Table 1 shows the details of the demographic data.

TABLE I. DEMOGRAPHIC DATA

\begin{tabular}{|c|c|c|c|}
\hline & & Number & $(\%)$ \\
\hline \multirow[t]{5}{*}{ Age group } & $20-29$ & 12 & $(2.9)$ \\
\hline & $30-39$ & 115 & $(27.8)$ \\
\hline & $40-49$ & 193 & $(46.7)$ \\
\hline & $50-59$ & 90 & (21.8) \\
\hline & 60 or above & 3 & $(0.7)$ \\
\hline \multirow[t]{2}{*}{ Gender } & Female & 300 & $(73.0)$ \\
\hline & Male & 113 & $(27.0)$ \\
\hline \multirow[t]{5}{*}{ Education } & Primary & 37 & $(9.0)$ \\
\hline & Secondary & 204 & $(49.4)$ \\
\hline & Associate degree & 71 & $(17.2)$ \\
\hline & Bachelor degree & 85 & $(20.6)$ \\
\hline & Master degree & 16 & $(3.9)$ \\
\hline \multirow{6}{*}{$\begin{array}{l}\text { Monthly } \\
\text { income (HK\$) }\end{array}$} & $<9,999$ & 33 & $(8.0)$ \\
\hline & $10,000-19,999$ & 94 & $(22.8)$ \\
\hline & $20,000-39,999$ & 190 & $(46.0)$ \\
\hline & $40,000-59,999$ & 85 & $(20.6)$ \\
\hline & $60,000-79,999$ & 7 & $(1.7)$ \\
\hline & 80,000 or above & 4 & $(1.0)$ \\
\hline
\end{tabular}




\section{B. HPV knowledge and barriers that affecting the willingness of vaccination}

Only $83 \%$ of participants had heard of HPV. The highest knowledge score was 7 marks. Only 18 respondents $(4.4 \%)$ scored 6 marks. $86(20.8 \%)$ respondents scored 0 , and 85 (20.6\%) scored 3 marks. Fig. 1 presents the detail. For individual questions about the HPV knowledge, around 19\% to $50 \%$ of parents answered correctly. Table II shows the percentage of correct answers scored by the parents.

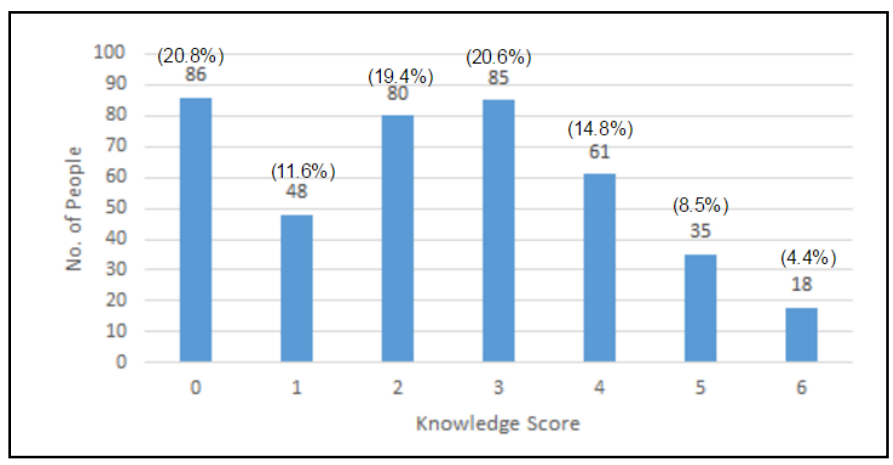

Fig. 1 Parental knowledge towards HPV

TABLE II. HPV KNOWLEDGE AND BARRIERS

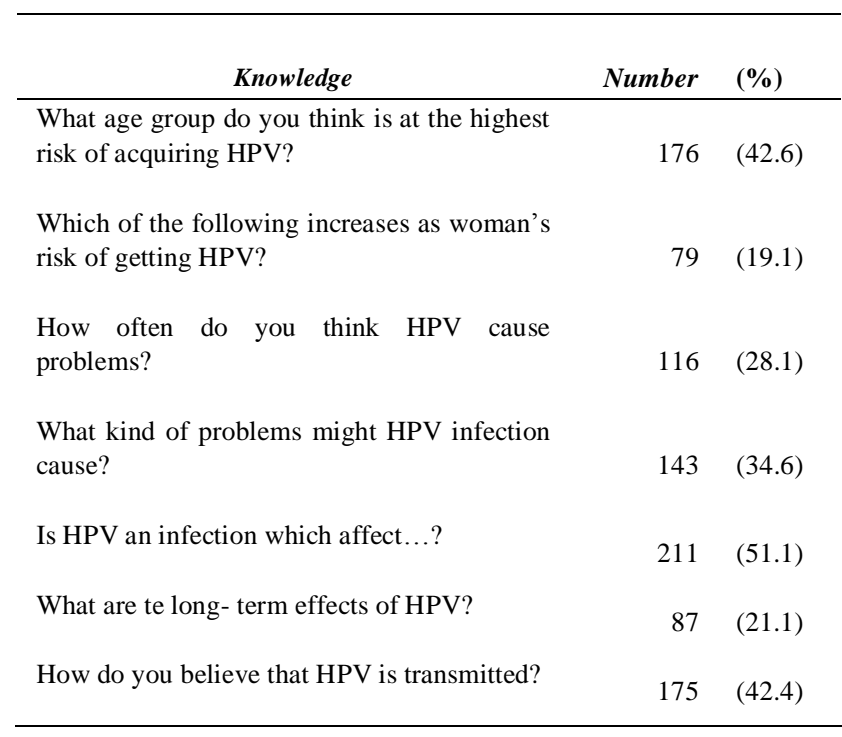

Around $36 \%$ of parents knew the price of HPV vaccine, and $72 \%$ thought that the cost is acceptable and worth to take after knowing the cost. The main reasons for not having their daughter(s) vaccinated were their worry about the side effects (86\%) and concern that the HPV vaccination would weaken the immune system of receivers $(38.5 \%)$. Moreover, $18 \%$ of parents believed that vaccination would induce early sexual activity or unprotected sex. Sources of information about HPV vaccine were from television (43.3\%), internet $(19.6 \%)$, doctors $(18.6 \%)$ and magazines $(16.9 \%)$. On a 1 to 10 scale measuring their willingness to have their daughters vaccinated, $69.8 \%$ parents indicated 6 or higher and $30.2 \%$ indicated 5 or below, meaning that more than half of the respondents were willing to vaccinate their daughter(s).

\section{Association between knowledge of HPV vaccine and willingness toward vaccination}

There was positive relationship between the knowledge of HPV among parents and their willingness toward vaccination; the correlation coefficient was 0.287 ( $\mathrm{p}<0.001$ ).

\section{DISCUSSION}

This study aimed to explore the factors influencing parental decisions on HPV vaccination for their daughters. The results of this study showed that knowledge of HPV vaccine and HPV infection was low in Hong Kong Chinese parents, as a majority of parents scored relatively low scores (0 marks, 20.8\%; 3 marks, 20.6\%), with an average score of 2.37. This result is consistent with the findings of previous studies conducted among Asian women [14] and university students [15]. This low score was rather unusual in this sample who were relatively highly educated. It may reflect the failure of HPV vaccination promotion strategies in our local community. Parents in this study were concerned about the side effects of HPV vaccine and held misbeliefs that HPV vaccination would have ill-effects on the receivers' immune system [16, 17]. This means that widespread education of parents about the effects, efficacy, and benefits of vaccination is lacking. As parental knowledge of HPV vaccine is positively related to their willingness toward vaccination [10, 11]. Health care professionals should provide needed information to parents to promote the vaccination rates.

For young females, it has been reported that parental perception that HPV vaccination will promote sexual activity is a significant barrier to vaccination $[10,18,19]$. Parents in this study also felt that vaccination at an early age would encourage earlier sexual activity or unprotected sex. It may due to the fact that they have difficulty to accept the sexual nature of HPV infections and the fact that their daughters are not old enough to initiate sexual activity [20].

To increase the vaccination rates for minors, perceived barriers in parents should be eliminated as parental decision 
affects initiation and completion of the vaccination [21]. Previous studies showed that the message provided by health care providers influences the decision of parents [22], and recommendations [23] and feedbacks [24] from health care providers indeed can boost the vaccination rate,]. Therefore, an effort should be made to convey clear message to parents about the high risk of HPV infection, the prevalence of cervical cancers associated with HPV infection, and the importance of vaccinating their daughters in early adolescence, before the individual has become sexually active.

Several limitations of this study must be noted. Firstly, this cross-sectional study recruited participants from four districts which might not represent the characteristics of all Hong Kong parents. This questionnaire elicited response from only one parent of a family; this may undermine the results as decision making in HPV vaccination for daughters could be a decision which involves both parents.

\section{CONCLUSION}

The present study confirms that the general knowledge about HPV vaccine and HPV associated knowledge is limited and may affect vaccine acceptability among Hong Kong parents. The findings of this study suggest that parental understanding about HPV vaccine affects their willingness to get their daughters vaccinated. Information about risks associated with HPV infection and the benefits of prophylactic HPV vaccines should be clear to parents. Education targeted towards parents will play a key role in the uptake of the HPV vaccination in young girls.

\section{ACKNOWLEDGMENTS}

The researchers would like to thank the parents who participated in this study.

\section{REFERENCES}

[1] Centers for Disease Control and Prevention. (2017). Genital HPV Infection - Fact Sheet. Centers for Disease Control and Prevention, U.S. Department of Health \& Human Services. Retrieved 15 April, 2017 from: https://www.cdc.gov/std/hpv/stdfact-hpv.htm

[2] Castellsagué, X. (2008). Natural history and epidemiology of HPV infection and cervical cancer.Gynecologic oncology, 110 (3 Suppl 2):S47. doi: 10.1016/j.ygyno.2008.07.045.

[3] Baseman, J. G., \& Koutsky, L. A. (2005). The epidemiology of human papillomavirus infections. Journal of Clinical Virology, 32, 16-24.

[4] Centre for Health Protection Department. (2013). Recommendation on the Use of Human Papillomavirus (HPV) Vaccine. Centre for Health Protection Department of Health, Hong Kong SAR. Retrieved 15 April, 2017 from: http://www.chp.gov.hk/files/pdf/recommendation_on_the_use_of_huma n_papillomavirus_hpv_vaccine_r.pdf

[5] Centers for Disease Control and Prevention. (2017). HPV Vaccine Information For Young Women. Centers for Disease Control and Prevention, U.S. Department of Health \& Human Services. Retrieved 15 April, 2017 from: https://www.cdc.gov/std/hpv/STDFact-HPVvaccine-young-women.htm

[6] Garland, S. M., Kjaer, S. K., Muñoz, N., Block, S. L., Brown, D. R., DiNubile, M. J., \& et al. (2016). Impact and effectiveness of the
Quadrivalent human Papillomavirus vaccine: A systematic review of 10 years of real-world experience. Lin Infect Dis, 63(4), 519-27.

[7] Schwarz, T. F. (2009). Clinical update of the AS04-Adjuvanted human Papillomavirus-16/18 cervical cancer vaccine, cervarix ${ }^{\circledR}$. Advances in Therapy, 26(11), 983-998. doi:10.1007/s12325-009-0079-5

[8] Department of Health. (2016). Cervical screening Programme - human Papillomavirus and vaccine. Department of Health, Hong Kong SAR. $\begin{array}{lllll}\text { Retrieved } & 15 & \text { April, } & 2017 & \text { from: }\end{array}$ http://www.cervicalscreening.gov.hk/english/hum/hum_ccv.html

[9] Li, S., Lau, Y., Lam, T., Yip, P., Fan, S., \& Ip, P. (2013). HPV vaccination in Hong Kong: Uptake and reasons for non-vaccination amongst Chinese adolescent girls. Vaccine, 31(49), 5785-8

[10] Allen, J. D., Coronado, G. D., Williams, R. S., Glenn, B., Escoffery, C., Fernandez, M., \& et al. (2010). A systematic review of measures used in studies of human Papillomavirus (HPV) vaccine acceptability. Vaccine, 28(24), 4027-37.

[11] Chan, S., Cheung, T., Lo, W., \& Chung, T. (2007). Women's attitudes on human papillomavirus vaccination to their daughters. The Journal of adolescent health : official publication of the Society for Adolescent Medicine, 41(2), 204-7.

[12] Census and Statistics Department. (2016). Population. Census and Statistics Department. Hong Kong SAR. Retrieved 15 February, 2017 from: http://www.censtatd.gov.hk/hkstat/sub/so20.jsp

[13] Munsell, D., Gray, C., Reed, J., Vasquez, J., \& Vlasak, J. (2009). A study of women and mothers'- of- minor- age- daughters knowledge of HPV as well as the attitudes toward the HPV vaccine. The Internet Journal of Academic Physician Assistants, 7(2)

[14] Wong, L. (2011). Knowledge and Attitudes About HPV Infection, HPV Vaccination, and Cervical Cancer Among Rural Southeast Asian Women. International Journal Of Behavioral Medicine, 18(2), 105-111. doi:10.1007/s12529-010-9104-y

[15] Chiang, V. C. L., Wong, H. T., Yeung, P. C. A., Choi, Y. K., Fok, M. S. Y., Mak, O. I., ... Wong, E. Y. Y. (2016). Attitude, Acceptability and Knowledge of HPV Vaccination among Local University Students in Hong Kong. International journal of environmental research and public health, 13(5), 486.

[16] Krawczyk, A., Knäuper, B., Gilca, V., Dubé, E., Perez, S., JoyalDesmarais, K. \& Rosberger, Z. (2015). Parents' decision-making about the human papillomavirus vaccine for their daughters: I. Quantitative results. Human Vaccines \& Immunotherapeutics, 11(2), 322-9.

[17] Voidăzan, S., Tarcea, M., Morariu, S. H., Grigore, A., \& Dobreanu, M. (2016). Human Papillomavirus Vaccine-Knowledge and Attitudes among Parents of Children Aged 10-14 Years: a Cross-sectional Study, Tirgu Mures, Romania. Central European journal of public health, 24(1), 29-38.

[18] Hendry, M., Lewis, R., Clements, A., Damery, S., \& Wilkinson, C. (2013). "HPV? Never heard of it!": A systematic review of girls' and parents' information needs, views and preferences about human papillomavirus vaccination. Vaccine, 31(45), 5152-5167.

[19] Koshel, C., Naganathan, S., \& Ballance, C. (2018). Improving Rates of Hpv Vaccination Among Female Patients in a Pediatric Resident Continuity Clinic.A significant barrier to vaccination is the parental perception that HPV vaccination promotes sexual activity

[20] Gonik, B. (2006). Strategies for Fostering HPV Vaccine Acceptance. Infectious Diseases in Obstetrics and Gynecology, 2006, 36797. http://doi.org/10.1155/IDOG/2006/36797

[21] Rutten, L. J. F., Sauver, J. L. S., Beebe, T. J., Wilson, P. M., Jacobson, D. J., Fan, C., ... \& Jacobson, R. M. (2017). Clinician knowledge, clinician barriers, and perceived parental barriers regarding human papillomavirus vaccination: Association with initiation and completion rates. Vaccine, 35(1), 164-169.

[22] Clark, S. J., Cowan, A. E., Filipp, S. L., Fisher, A. M., \& Stokley, S. (2016). Parent perception of provider interactions influences HPV vaccination status of adolescent females. Clinical pediatrics, 55(8), 701 706.

[23] Daly, K. L., Halon, P. A., Aronowitz, T., \& Ross, G. (2016). A university health initiative to increase human papillomavirus vaccination rates. The Journal for Nurse Practitioners, 12(6), e281-e286. 
[24] Rand, C. M., Schaffer, S. J., Dhepyasuwan, N., Blumkin, A., Albertin, C., Serwint, J. R.,\& et al. (2018). Provider communication, prompts, and feedback to improve HPV vaccination rates in resident clinics. Pediatrics, e20170498. 